\title{
Relationship of Hair Cortisol with History of Psychosis, Neuropsychological Performance and Functioning in Remitted Later-Life Major Depression
}

\author{
Kathleen S. Bingham ${ }^{a, b}$ Benoit H. Mulsant ${ }^{a, c}$ Deirdre R. Dawson ${ }^{d, e}$ \\ Samprit Banerjee ${ }^{f} \quad$ Alastair J. Flint ${ }^{a, b}$ \\ a Department of Psychiatry, University of Toronto, Toronto, ON, Canada; ${ }^{b}$ Centre for Mental Health, University Health \\ Network, Toronto, ON, Canada; ${ }^{C}$ Centre for Addiction and Mental Health, Toronto, ON, Canada; ${ }^{\mathrm{d} D e p a r t m e n t}$ of \\ Occupational Science \& Occupational Therapy, University of Toronto, Toronto, ON, Canada; ${ }^{e}$ Rotman Research \\ Institute, Toronto, ON, Canada; 'Department of Biostatistics and Epidemiology, Weill Cornell Medical College, \\ New York, NY, USA
}

\section{Keywords}

Major depression · Psychotic depression .

Cognitive performance $\cdot$ Cortisol · Hair cortisol .

Hypothalamic-pituitary-adrenal axis

\begin{abstract}
Introduction: Major depressive disorder (MDD) is associated with hypothalamic-pituitary-adrenal axis dysfunction that may persist into remission. Preliminary evidence suggests that this dysfunction may be associated with impaired neuropsychological performance in remitted MDD. MDD with psychotic features ("psychotic depression") is associated with greater neuropsychological and functional impairment than nonpsychotic depression, including in remission. Therefore, the aim of this exploratory study was to examine the relationships among hair cortisol concentration (HCC) - a marker of longer term endogenous cortisol exposure - and history of psychotic features, neuropsychological performance, and functioning in remitted MDD. Methods: This cross-sectional study compared the relationship between HCC and (i) history of psychosis, (ii) neuropsychological performance, and (iii) everyday functioning in a
\end{abstract}

karger@karger.com www.karger.com/nps

Karger"

BOPEN ACCESS (c) 2021 The Author(s)

Published by S. Karger AG, Base

This is an Open Access article licensed under the Creative Commons Attribution-NonCommercial-4.0 International License (CC BY-NC) (http://www.karger.com/Services/OpenAccessLicense), applicable to the online version of the article only. Usage and distribution for commercial purposes requires written permission. group of 60 participants with remitted later-life MDD using Pearson's correlation coefficients. This study also measured HCC in a group of 36 nonpsychiatric volunteers to examine the clinical significance of HCC in the patient group. $\boldsymbol{R e}$ sults: There were no statistically significant correlations between HCC and history of psychotic features, neuropsychological performance, or functioning. Furthermore, there was no clinically meaningful difference in $\mathrm{HCC}$ between patients and nonpsychiatric volunteers. Conclusion: This study is the first to examine HCC in psychotic depression. The results do not support the hypothesis that impaired neuropsychological performance, and everyday function in remitted psychotic depression is due to a sustained elevation of cortisol.

(c) 2021 The Author(s)

Published by S. Karger AG, Basel

\section{Introduction}

Several decades of research have suggested that the hypothalamic-pituitary-adrenal (HPA) axis is implicated in a variety of disorders with affective and cognitive symptoms (Keller et al. [1]). Up to $40-60 \%$ of patients with 
major depressive disorder (MDD) demonstrate hypercortisolemia (a marker of HPA axis dysregulation) during an acute episode [1]. Although HPA axis dysregulation was historically conceptualized as a state phenomenon in MDD that resolved with remission of the depression [2], some studies have reported elevated blood and salivary cortisol levels in patients with remitted MDD compared to healthy controls $[3,4]$. These findings, however, have not been consistent across studies: other authors have not identified a difference in cortisol levels [5] or response to the dexamethasone- / corticotropin-releasing hormone (Dex/CRH) test $[6,7]$ between patients with remitted MDD and controls. These discrepant results in remitted depression may be due to heterogeneity in study populations. None of these studies focused on patients with remitted psychotic major depression (PMD), a group that has more pronounced HPA axis dysregulation than nonpsychotic major depression (NPMD) during acute depressive episodes [8].

Elevated cortisol has been found to be associated with cognitive dysfunction in a variety of conditions, including MDD [6,9-12]. Studies evaluating the link between cortisol and cognition in MDD have mostly focused on patients with acute depression. The limited evidence regarding the relationship between HPA axis function and neuropsychological performance in remitted depression is mixed. Reppermund et al. [13] found that although a high proportion of depressed inpatients remained cognitively impaired despite improvement in symptom severity, the cortisol response to the Dex/CRH test was not related to cognitive impairment in remitted patients. On the other hand, Behnken et al. [6] found significant associations between cortisol response to the Dex/CRH test and verbal memory, attention, cognitive speed, and executive function in patients with remitted MDD. The same relationships were not observed in a nonpsychiatric control group, leading the authors to suggest that patients with MDD may be more vulnerable to subtle dysregulations of their HPA axis. The relationship between HPA axis dysregulation and everyday functioning in MDD has only been examined by one study that demonstrated a negative relationship between 24 -h urinary cortisol levels and social functioning independent of depressive symptom severity in a group including both PMD and NPMD patients followed prospectively for 1 year [14].

PMD is a severe type of MDD, i.e., more prevalent in older adults [15]. It is associated with poorer neuropsychological performance and poorer everyday functioning than NPMD, in both acute and remitted states [16-18].
As mentioned above, it is associated with more pronounced HPA axis dysregulation. These findings raise the question as to whether HPA axis dysregulation contributes to the neuropsychological and functional impairment in remitted PMD.

Therefore, we conducted this exploratory study to examine the association of HPA axis function in patients with remitted MDD with: (i) history of psychotic features, (ii) neuropsychological performance, and (iii) everyday functioning. The HPA axis function was also examined in a nonpsychiatric group to place the results in clinical context. This study focused on patients in remission, so as to control for the confounding effect of symptom severity on neuropsychological performance and functioning. Finally, to explore whether there is a relationship between sustained HPA dysfunction in remission and the aforementioned variables, we measured hair cortisol concentration (HCC) because it is a measure of longer term cortisol exposure.

\section{Materials and Methods}

\section{Study Design and Procedures}

This was a cross-sectional observational study. Using procedures approved by the local institutional review board, written informed consent was obtained from all participants prior to the initiation of study procedures. Data were collected in one assessment by a single rater (K.S.B.) who was blind to patient-participants' history of psychotic features and current medications until the end of the assessment, at which point data pertaining to history of psychotic features and current medications were obtained.

\section{Participants}

As reported previously, patient participants were recruited from psychiatric outpatient clinics of 2 academic hospitals in Toronto, Canada. We focused on adults 50 years or older only because PMD is a more stable diagnosis later in life. Inclusion criteria were (1) 50 years or older; (2) a history of MDD according to the criteria of the Diagnostic and Statistical Manual of Mental Disorders, fifth edition (DSM-5) [20], with the most recent episode within the past 10 years; (3) in remission, as defined in the DSM-5 (i.e., not meeting DSM-5 criteria for a major depressive episode or psychosis) for a minimum of 4 weeks at the time of assessment; (4) a 17-item Hamilton Depression Rating Scale (HDRS) [21] score $\leq 10$ and Schedule for Affective Disorders and Schizophrenia (SADS) [22] delusional and hallucination item scores of 1 (i.e., absence of psychosis) at the time of assessment. Exclusion criteria were (1) inability to communicate in English; (2) meeting DSM-5 criteria for (a) a major neurocognitive disorder; (b) another current psychiatric disorder, except specific phobia; (c) a lifetime diagnosis of schizophrenia, schizoaffective disorder, delusional disorder, bipolar disorder, intellectual disability, learning disorder, attention deficit hyperactivity disorder, obsessive-compulsive disorder, or autism spectrum disorder; (3) physical disability or sensory impairment severe enough to prevent participation in the as- 
sessment; (4) any medical or neurologic disorder severely impacting functioning or cognition (e.g., Parkinson's disease, multiple sclerosis); (5) lifetime diagnosis of a traumatic or acquired brain injury resulting in permanent neurologic impairment; (6) current active seizure disorder; and (7) treatment with electroconvulsive therapy in the preceding 6 months.

The nonpsychiatric group included volunteers aged 50 years or older who were matched by age-group (by decade) and gender to the patient participants. Inclusion criteria were (1) age 50 years or older; (2) a 17-item HAM-D total score $\leq 10$ and SADS delusional and hallucination item scores of 1 (absence of psychosis). Exclusion criteria were the same as for patient participants, except that in addition the nonpsychiatric group were required to have no lifetime history of MDD.

All participants provided written informed consent. This study protocol was approved by the Research Ethics Board at the study site.

\section{Measurement of Cortisol}

An emerging technique for measuring longer term exposure to cortisol is the analysis of hair cortisol levels. There are several benefits to this technique. One is the ability to measure average activity of the HPA axis over a specified time period, since hair typically grows about $1 \mathrm{~cm}$ per month [23]. HCC has been validated as a biomarker of longer term exposure to endogenous cortisol by demonstrating elevated levels in conditions known to be associated with elevated cortisol, including Cushing's disease and pregnancy [24]. Further HCC has been positively correlated with 30 -day integrated salivary cortisol area under the curve in healthy adults [25]. Because cortisol concentration may not be consistent along the hair shaft due to factors such as environmental exposure, it is recommended that hair cortisol analyses be restricted to the most proximal 5-6 $\mathrm{cm}$ of hair, as this segment has been demonstrated to reliably correlate with systemic cortisol exposure $[26,27]$.

The hair collection procedure was based on expert recommendation as follows: (1) using scissors, hair was lifted from the vertex posterior of the scalp to clear an area for sampling; (2) a sliver of hair (approximately 75 strands) was selected and cut in a straight horizontal line, as close to the scalp as possible; (3) the hair was placed on aluminum foil and secured with painters' tape. The foil was then folded and placed in an envelope. Samples were labeled with participants' study ID numbers and the date. Hair samples were stored at room temperature prior to delivery to the University of Colorado Behavioral Immunology and Endocrinology laboratory. Participants were given a brief questionnaire to document their hair washing frequency and cosmetic treatment habits. Variables included frequency of hair washing and conditioning, presence of hair straightening, and use of hair coloring, bleaching, and perm treatments. These variables are known to impact HCC [27] and were, therefore, compared between PMD and NPMD participants.

Cortisol concentrations were determined from the first $3-\mathrm{cm}$ hair segment most proximal to the scalp or from as much as was available for participants with shorter hair. Based on an average hair growth of $1 \mathrm{~cm} /$ month [27], this hair segment is assumed to represent the hair growth over the 3-month period prior to the sampling. If participants had been in symptomatic remission for $<3$ months, HCCs were analyzed only for the segments of hair representing the time period of remission. Cortisol was extracted from hair samples, and cortisol concentration was measured using the validated high-sensitivity enzyme immunoassay described by Lafferty et al. [28]. Using this method, coefficients of variation (compared to internal laboratory control samples) have been reported to be $1.9 \%$ for intra-assay variability and $11.6 \%$ for inter-assay variability [28].

\section{Clinical and Neuropsychological Measures}

History of psychotic features was assessed using the Structured Clinical Interview for DSM-5, research version (SCID-5-RV) [29], supplemented by collateral information obtained from medical records after the SCID was administered. Two measures were used to assess everyday functioning: (i) total number of cues from the Performance Assessment of Self-Care Skills (PASS) [30,31] modified such that each cue is weighted according to the level of assistance required (higher scores indicate more impairment) and (ii) frequency scores from the Late-Life Function and Disability Instrument (LLFDI), disability component [32] (lower scores indicate more impairment). The PASS, a performance-based instrument, measures instrumental activity of daily living performance by providing participants with a hierarchical series of cues when they are performing a series of instrumental activity of daily living tasks (e.g., verbal prompts, physical assistance). Higher numbers of cues mean less ability to perform tasks independently. The PASS has been shown to be sensitive to disability in participants with remitted late-life depression [30]. The LLFDI, a participant report instrument, measures general functioning. It has been shown to be associated with clinical measures in participants with late-life depression and to be sensitive to improvement in depressive symptoms [33].

The measures of neuropsychological performance pertained to the domains of processing speed, executive function, and verbal memory and were assessed using subtests from 2 batteries: the Delis-Kaplan Executive Functioning System (DKEFS) [34] and the Repeatable Battery for the Assessment of Neuropsychological Status (RBANS) [35]. Specific subtests included were the DKEFS Trail Making Test, condition 5 (motor speed); DKEFS Color Naming Test (simple processing speed); RBANS Coding (complex psychomotor speed); DKEFS Color-Word Interference Test (executive function-inhibition); DKEFS Trail Making Test, condition 4 versus condition 5 (executive function-task switching); RBANS List Learning (immediate verbal memory) and the RBANS List Recall (delayed verbal memory). The neuropsychological domains were selected based on literature suggesting that verbal memory, processing speed, and executive functions are most affected in laterlife depression, specifically in the euthymic state [36]. The RBANS and DKEFS have both been shown to be sensitive to cognitive deficits in late-life depression $[37,38]$. In addition to age, gender, race, education, and total MMSE score [39], several additional variables were examined based on the evidence that they may have a clinically significant impact on HCC (smoking status, hair washing frequency, and use of hair dye) [27] or that they may have a relationship with HCC and are clinically likely to be associated with history of PMD (use of antipsychotic and antidepressant medication, burden for physical illness measured using the Cumulative Illness Rating Scale for Geriatrics (CIRS-G) [40], and depressive symptoms, measured using the HDRS).

\section{Data Analytic Plan}

In order to examine the relationship of HCC with (i) history of psychotic features, (ii) neuropsychological performance, and (iii) everyday functioning, we performed 3 sets of Pearson's prod- 
Table 1. Characteristics of participants with MDD in remission $(N=60)$

\begin{tabular}{lc}
\hline Variable & \\
\hline Age, years, mean (SD) & $69.5(8.0)$ \\
Gender, $N$ (\%) & \\
$\quad$ Female & $38(63.3)$ \\
$\quad$ Male & $22(36.67)$ \\
Race, $N$ (\%) & \\
$\quad$ White & $51(85.0)$ \\
$\quad$ Black & $4(6.7)$ \\
$\quad$ Asian & $5(8.3)$ \\
Education (standardized years), mean (SD) & $15.1(3.1)$ \\
History of psychosis, $N(\%)$ & $17(28.3)$ \\
HDRS total score, mean (SD) & $4.0(2.9)$ \\
CIRS-G total score, mean (SD) & $6.3(4.2)$ \\
Diabetes, $N$ (\%) & $7(11.7)$ \\
Vascular disease (hypertension, & \\
$\quad$ atherosclerosis), $N(\%)$ & $25(41.7)$ \\
Heart disease, $N(\%)$ & $16(26.7)$ \\
Active cancer, $N(\%)$ & $0(0)$ \\
Rheumatic disease, $N(\%)$ & $3(5)$ \\
MMSE total score, mean (SD) & $28.6(1.6)$ \\
Duration of remission (months), mean (SD) & $13.1(21.7)$ \\
Current antidepressant use, $N(\%)$ & $51(85.0)$ \\
Current antipsychotic use, $N(\%)$ & $16(26.7)$ \\
Current corticosteroid use, $N(\%)$ & $0(0)$ \\
Current Smoker, $N(\%)$ & $2(3.3)$ \\
Hair wash frequency, $N(\%)$ & $19(31.7)$ \\
$\quad 1 \times /$ week & $30(50.0)$ \\
$\quad>4 \times /$ week & $11(18.3)$ \\
Use of hair dye & $26(45.6)$ \\
\hline & \\
& \\
& \\
&
\end{tabular}

CIRS-G, Cumulative Illness Rating Scale for Geriatrics; HDRS, Hamilton Depression Rating Scale; MMSE, Mini-Mental State Examination; MDD, major depressive disorder.

Table 2. Correlations (Pearson's $r$ ) between HCC and neuropsychological performance $(N=60)$

\begin{tabular}{lccl}
\hline $\begin{array}{l}\text { Neuropsychological } \\
\text { variable }\end{array}$ & $\begin{array}{l}\text { Correlation }(r) \\
\text { with HCC }\end{array}$ & df & $p$ value \\
\hline TMT5 $^{1}$ & -0.07 & 58 & 0.58 \\
Color naming & 0.02 & 58 & 0.87 \\
Coding & 0.02 & 58 & 0.9 \\
CWI & -0.05 & 58 & 0.71 \\
TMT4 versus 5 & -0.05 & 58 & 0.73 \\
List learning & 0.2 & 58 & 0.13 \\
List recall & 0.1 & 58 & 0.45 \\
\hline
\end{tabular}

HCC, hair cortisol concentration. ${ }^{1}$ Transformed variable used in analysis due to skewness. uct-moment correlation analyses. Given the exploratory nature of these analyses, we did not correct for multiple comparisons. The HCC variable was highly skewed, and we used a logarithmic transformation for all analyses. The following variables were also transformed due to skewness: PASS score (logarithmic transformation), TMT5 (cubic polynomial transformation), and TMT condition 4 versus 5 (square root transformation). In the event of a statistically significant correlation between HCC and a variable of interest, we planned to perform linear regression analysis controlling for relevant sociodemographic and clinical variables.

To compare HCC results between patient and nonpsychiatric groups, we calculated effect sizes and their $95 \%$ confidence limits. To compare the variation in HCC among the NPMD, PMD, and nonpsychiatric groups, we examined the interquartile ranges (IQRs) of the raw HCC scores of the 3 groups using boxplot. We set boxplot whiskers to demonstrate the lowest data point within 1.5 IQR of the lower quartile and the highest data point within 1.5 IQR of the upper quartile. Since the nonpsychiatric group was solely used to generate reference data to interpret the clinical importance of the patients' HCC data, we did not perform correlational analyses on the data from the nonpsychiatric control participants.

\section{Results}

\section{Description of Participants and Hair Samples}

Sixty patients (17 with PMD and 43 with NPMD) and 37 nonpsychiatric volunteers participated and provided a hair sample for cortisol analysis. One hair sample from the nonpsychiatric group could not be analyzed due to an insufficient sample weight. This left 36 hair samples from the nonpsychiatric group available for analysis. Eight of the patient samples (all NPMD) and one of the control samples were below the weight $(5 \mathrm{mg}$ ) recommended for the inter-assay and intra-assay reliability cited in the Methods section. As such, the potential assay error is higher (+15-25\%). Further, for one additional healthy volunteer participant, the HCC results $(1.5 \mathrm{pg} / \mathrm{mg})$ were lower than the sensitivity for the assay. As such, we performed a sensitivity analysis excluding participants with potentially less reliable results. Characteristics of the patient participants are shown in Table 1, including sociodemographic and clinical data. Of the 17 participants with PMD, 6 had mood-incongruent delusions (not shown in Table).

\section{Relationship between History of Psychotic Features and Hair Cortisol Concentration in Remitted Later- \\ Life Major Depressive Disorder}

The NPMD and PMD participants had a mean (SD) HCC of 18.86 (32.97) and 12.48 (11.26) pg/mg, respectively. The correlation ( $\mathrm{df}$ ) between history of psychotic features and HCC was $r(58)=-0.02, p=0.88$. 
Table 3. Cohen's D effect sizes for the difference in HCC between PMD and NPMD groups and the nonpsychiatric group

\begin{tabular}{lllll}
\hline $\begin{array}{l}\text { Non-Psych, } \\
N=36\end{array}$ & $\begin{array}{l}\text { NPMD, } \\
N=43\end{array}$ & $\begin{array}{l}\text { Effect size }^{1} \text { (95\% CL) } \\
\text { (Non-Psych. vs. NPMD) }\end{array}$ & $\begin{array}{l}\text { PMD, } \\
\text { N=17 }\end{array}$ & $\begin{array}{l}\text { Effect size }^{1} \text { (95\% CL) } \\
\text { (Non-Psych. vs. PMD) }\end{array}$ \\
\hline $\begin{array}{l}H C C, p g / m g \\
14.56(20.43)\end{array}$ & $18.86(32.9)$ & $0.26(0.21,0.31)$ & $12.48(11.3)$ & $0.25(0.16,0.34)$ \\
\hline
\end{tabular}

HCC, hair cortisol concentration; NPMD, nonpsychotic major depression; PMD, psychotic major depression; non-Psych, nonpsychiatric comparison group. ${ }^{1}$ Logarithmic transformation used for analysis due to right skewness.

\section{Relationship between Hair Cortisol Concentration and Neuropsychological Performance in Remitted Later-Life Major Depressive Disorder}

The results of the correlation analyses examining the relationship of HCC with each of the neuropsychological measures are shown in Table 2. None of the correlations were statistically significant.

Relationship between Hair Cortisol Concentration and Functioning in Remitted Later-Life Major Depressive Disorder

The correlation (df) of HCC with PASS was $r(58)=$ $-0.04(p=0.78)$, and HCC with LLFDI was $r(58)=-0.07$ $(p=0.57)$.

\section{Magnitude of Difference in Mean Hair Cortisol \\ Concentration between Patient and Nonpsychiatric \\ Groups}

The effect sizes for the magnitude of the difference in HCC between each patient group and the nonpsychiatric group are shown in Table 3. The effect sizes were small. Figure 1 shows the distribution of HCC among the 3 groups and a comparison of the distribution of HCC. There is substantial overlap in the distribution of HCC among all 3 groups.

\section{Sensitivity Analyses}

All analyses were repeated after excluding the 8 NPMD and 2 healthy volunteer participants for whom the assay reliability was potentially less. None of the results were qualitatively different and all remained statistically insignificant.

\section{Discussion/Conclusion}

To our knowledge, this is the first study to assess HCC in PMD. We did not find a significant relationship between history of psychotic features and 3-month HCC in

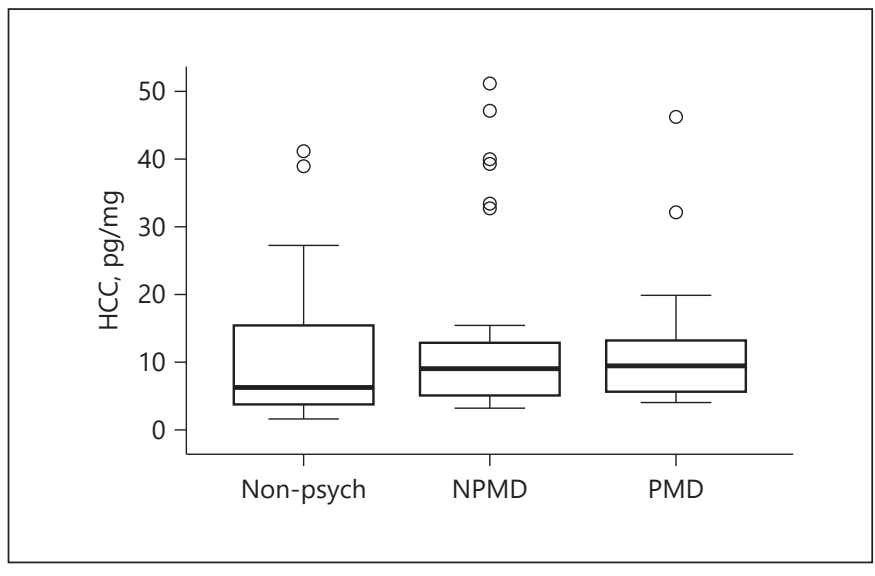

Fig. 1. Boxplots depicting 1.5 IQR for HCCs in each participant group. Whiskers demonstrate the lowest data point within $1.5 \mathrm{IQR}$ of the lower quartile and the highest data point within $1.5 \mathrm{IQR}$ of the upper quartile. Note that 2 additional outliers in the NPMD group $(\mathrm{HCC}=127.71$ and $\mathrm{HCC}=179.28 \mathrm{pg} / \mathrm{mg})$ are not shown in the boxplot in order to visualize the data distribution clearly. HCC, hair cortisol concentration; Nonpsych, nonpsychiatric comparison group; NPMD, nonpsychotic major depression group; PMD, psychotic major depression group.

patients with later-life MDD in sustained remission. Moreover, HCC did not have a significant relationship with neuropsychological or functional measures in remitted MDD. Thus, these results do not support the hypothesis that sustained elevation of cortisol explains the worse neuropsychological performance and everyday function in remitted PMD.

The negative findings of this study could reflect a true null effect. This conclusion is supported by the absence of a clinically important difference in mean HCC between the patient groups and an age- and sex-matched nonpsychiatric group, as well as the very weak correlation coefficients between HCC and the variables of interest. 
However, other factors need to be considered. First, it is possible that the negative findings were the result of a type II error due to inadequate sample size. A sample size of 85 participants is required to find a weak correlation of 0.3 , at a 2-tailed alpha level of 0.05 and a beta level of 0.2 [41]. Thus, it is possible that our study was under-powered to detect an actual small to moderate difference in HCC between PMD and NPMD patients and that a stronger correlation coefficient might be found with a larger sample size. However, given the very weak magnitude of the correlation coefficient observed between history of psychosis and HCC $(r=-0.02)$, it is unlikely that increasing our sample size by about one-third would result in a substantially different finding.

Generally, studies investigating HCC in current MDD have had mixed results, with findings that HCC is higher $[42,43]$ than, or the same [44-46], or lower [47] as healthy controls. Some of these studies reported on the influence of various additional clinical variables. One study found that, while there was no difference in HCC between patients and controls, childhood trauma in both groups was associated with lower HCC, suggesting that other clinical variables may be more relevant to HCC than presence of MDD [45]. Another study reported that patients with comorbid depressive and anxiety disorders had higher HCC than control participants, though patients with either depression or anxiety alone did not [44]. A third study found that, while there was no difference between HCC in recurrent MDD patients and controls, those with firstepisode MDD had higher HCC relative to both other groups [46]. This evidence suggests that MDD itself may not be associated with longer term endogenous cortisol exposure as measured by HCC, but that other associated clinical factors may play a role. Although HCC has not previously been studied in remitted MDD, the mixed evidence cited in the Introduction regarding blood and salivary cortisol measures in remitted MDD suggests that there may not be substantial HPA axis dysregulation in remitted MDD either.

Variable findings regarding cortisol in MDD may be related, in part, to differences in study populations. Our study included older adults with moderate levels of medical comorbidity (see Table 1) and associated medications, which can influence cortisol concentration outside of depression, potentially confounding the relationship between depression and hair cortisol. Comorbid medical issues may be less likely in younger populations.

There are several limitations to this study. First, the study was exploratory and the sample size was not based on a power calculation to test a specific hypothesis. Sec- ond, although HCC has the advantage of being low burden for participants and it can also give an indication of cortisol exposure over a prolonged period of time, findings regarding HCC in MDD have been less consistent than tests of HPA function that collect serial measures of cortisol. Third, because patients were in remission, the diagnosis of psychotic depression was based on their recall of the diagnosis or of having experienced psychotic features. Even though the details of patient interview were subsequently checked by chart review, it is possible that some cases of PMD were missed.

To conclude, the findings of this exploratory study do not support the hypothesis that impairment of neuropsychological performance or function is associated with elevation of cortisol in remitted PMD in older adults. If future studies test this hypothesis, it is recommended that they use measures of cortisol function other than HCC.

\section{Acknowledgements}

The authors wish to acknowledge Dr. Denise Chisholm for training and supervision regarding the PASS and Mr. Ola Kuforiji for assistance with data entry and cleaning, as well as Dr. Mark Laudenslager and his research staff from the University of Colorado Denver Behavioral Immunology and Endocrinology Laboratory for analysis of the HCC.

\section{Statement of Ethics}

This study complied with local guidelines for research involving human participants and was conducted ethically in accordance with the World Medical Association Declaration of Helsinki. This study was approved by the study site's Research Ethics Board, and all participants provided written informed consent.

\section{Conflict of Interest Statement}

K.S. Bingham receives research funding through the University of Toronto, Department of Psychiatry's Excellence Fund and the Labatt Family Network Catalyst grant. During the past 3 years, B.H. Mulsant has received research funding from Brain Canada, the CAMH Foundation, the Canadian Institutes of Health Research, and the US National Institutes of Health (NIH); research support from Bristol-Myers Squibb (medications for a NIH-funded clinical trial), Eli-Lilly (medications for a NIH-funded clinical trial), Pfizer (medications for a NIH-funded clinical trial), Capital Solution Design LLC (software used in a study funded by CAMH Foundation), and HAPPYneuron (software used in a study funded by Brain Canada). He directly owns stocks of General Electric (<USD 5,000). 
During the past 3 years, D.R. Dawson received grant support from the Canadian Institutes of Health Research and the Canadian Partnership for Stroke Recovery and within the past 3 years has received honoraria from the American Congress of Rehabilitation Medicine and Texas Women's University. S. Banerjee has no conflicts to declare. During the past 3 years, A.J. Flint received grant support from the US National Institutes of Health, the Patient-Centered Outcomes Research Institute, the Canadian Institutes of Health Research, Brain Canada, and the Alzheimer's Association.

\section{Funding Sources}

This study was funded by a grant from the University Health Network, Center for Mental Health.

\section{Author Contributions}

Dr. Bingham: conceptualization; data curation; formal analysis; funding acquisition; investigation; methodology; project administration; resources; software; visualization; roles/writing - original draft; writing - review and editing. Dr. Dawson: conceptualization; methodology; supervision; writing - review and editing. Dr. Mulsant: conceptualization; methodology; supervision; writing - review and editing. Dr. Banerjee: supervision (data analysis); supervision; writing - review and editing. Dr. Flint: conceptualization; data curation; funding acquisition; investigation; methodology; project administration; resources; supervision; software; writing - review and editing. All authors provided final approval of the manuscript and agree to be accountable for all aspects of the work in ensuring that questions related to the accuracy or integrity of any part of the work are appropriately investigated and resolved.

\section{References}

1 Keller J, Gomez R, Williams G, Lembke A, Lazzeroni L, Murphy GM, et al. HPA axis in major depression: cortisol, clinical symptomatology, and genetic variation predict cognition. Mol Psychiatry. 2017 Apr;22(4): 527-36.

2 Arana GW, Baldessarini RJ, Ornsteen M. The dexamethasone suppression test for diagnosis and prognosis in psychiatry. Commentary and review. Arch Gen Psychiatry. 1985 Dec; 42(12):1193-204.

3 Holsen LM, Lancaster K, Klibanski A, Whitfield-Gabrieli S, Cherkerzian S, Buka S, et al. HPA-axis hormone modulation of stress response circuitry activity in women with remitted major depression. Neuroscience. 2013 Oct:250:733-42.

4 Vreeburg SA, Hoogendijk WJ, van Pelt J, Derijk RH, Verhagen JC, van Dyck R, et al. Major depressive disorder and hypothalamic-pituitary-adrenal axis activity: results from a large cohort study. Arch Gen Psychiatry. 2009 Jun; 66(6):617-26.

5 Gerritsen L, Comijs HC, van der Graaf Y, Knoops AJ, Penninx BW, Geerlings MI. Depression, hypothalamic pituitary adrenal axis, and hippocampal and entorhinal cortex volumes: the SMART Medea study. Biol Psychiatry. 2011 Aug;70(4):373-80.

6 Behnken A, Bellingrath S, Symanczik JP, Rieck MJ, Zavorotnyy M, Domschke K, et al. Associations between cognitive performance and cortisol reaction to the DEX/CRH test in patients recovered from depression. Psychoneuroendocrinology. 2013 Mar;38(3):44754.

7 Ferguson EH, Di Florio A, Pearson B, Putnam KT, Girdler S, Rubinow DR, et al. HPA axis reactivity to pharmacologic and psychological stressors in euthymic women with histories of postpartum versus major depression. Arch Womens Ment Health. 2017 Jun;20(3): 411-20.

8 Keller J, Flores B, Gomez RG, Solvason HB, Kenna H, Williams GH, et al. Cortisol circa- dian rhythm alterations in psychotic major depression. Biol Psychiatry. 2006 Aug;60(3): 275-81.

9 Bourdeau I, Bard C, Noël B, Leclerc I, Cordeau MP, Bélair M, et al. Loss of brain volume in endogenous Cushing's syndrome and its reversibility after correction of hypercortisolism. J Clin Endocrinol Metab. 2002 May; 87(5):1949-54.

10 Egeland J, Lund A, Landrø NI, Rund BR, Sun$\operatorname{det} \mathrm{K}$, Asbjørnsen A, et al. Cortisol level predicts executive and memory function in depression, symptom level predicts psychomotor speed. Acta Psychiatr Scand. 2005 Dec; 112(6):434-41.

11 McLennan SN, Ihle A, Steudte-Schmiedgen S, Kirschbaum C, Kliegel M. Hair cortisol and cognitive performance in working age adults. Psychoneuroendocrinology. 2016 May;67: 100-3.

12 Rubinow DR, Post RM, Savard R, Gold PW. Cortisol hypersecretion and cognitive impairment in depression. Arch Gen Psychiatry. 1984 Mar;41(3):279-83.

13 Reppermund S, Zihl J, Lucae S, Horstmann S, Kloiber S, Holsboer F, et al. Persistent cognitive impairment in depression: the role of psychopathology and altered hypothalamic-pituitary-adrenocortical (HPA) system regulation. Biol Psychiatry. 2007 Sep;62(5):400-6.

14 Rothschild AJ, Samson JA, Bond TC, Luciana MM, Schildkraut JJ, Schatzberg AF. Hypothalamic-pituitary-adrenal axis activity and 1 -year outcome in depression. Biol Psychiatry. 1993 Sep;34(6):392-400.

15 Parker G, Hadzi-Pavlovic D, Hickie I, Mitchell P, Wilhelm K, Brodaty H, et al. Psychotic depression: a review and clinical experience. Aust N Z J Psychiatry. 1991 Jun;25(2):16980.

16 Bingham KS, Dawson DR, Mulsant BH, Banerjee S, Flint AJ. Relationships among history of psychosis, cognition and functioning in later-life remitted major depression. Am J Geriatr Psychiatry. 2020.
17 Coryell W, Leon A, Winokur G, Endicott J, Keller M, Akiskal H, et al. Importance of psychotic features to long-term course in major depressive disorder. Am J Psychiatry. 1996 Apr;153(4):483-9.

18 Fleming SK, Blasey C, Schatzberg AF. Neuropsychological correlates of psychotic features in major depressive disorders: a review and metaanalysis. J Psychiatr Res. 2004 Jan;38(1):27-35.

19 Martinez RA, Mulsant BH, Meyers BS, Lebowitz BD. Delusional and psychotic depression in late life clinical research needs. Am J Geriatr Psychiatry. 1996;4(1):77-84.

20 APA. Diagnostic and statistical manual of mental disorders. 5th ed. American Psychiatric Association; 2013.

21 Hamilton M. A rating scale for depression. J Neurol Neurosurg Psychiatry. 1960 Feb;23: 56-62.

22 Spitzer R, Endicott J. Schedule for Affective Disorders and Schizophrenia. 3rd ed. New York, NY: Biometrics Research Dept, New York State Psychiatric Institute; 1979.

23 Wennig R. Potential problems with the interpretation of hair analysis results. Forensic Sci Int. 2000 Jan; 107(1-3):5-12.

24 Gow R, Thomson S, Rieder M, Van Uum S, Koren G. An assessment of cortisol analysis in hair and its clinical applications. Forensic Sci Int. 2010 Mar;196(1-3):32-7.

25 Short SJ, Stalder T, Marceau K, Entringer S, Moog NK, Shirtcliff EA, et al. Correspondence between hair cortisol concentrations and 30-day integrated daily salivary and weekly urinary cortisol measures. Psychoneuroendocrinology. $2016 \mathrm{Sep} ; 71: 12-8$.

26 Gao W, Xie Q, Jin J, Qiao T, Wang H, Chen $\mathrm{L}$, et al. HPLC-FLU detection of cortisol distribution in human hair. Clin Biochem. 2010 May;43(7-8):677-82.

27 Russell E, Koren G, Rieder M, Van Uum S. Hair cortisol as a biological marker of chronic stress: current status, future directions and unanswered questions. Psychoneuroendocrinology. 2012 May;37(5):589-601. 
28 Lafferty DJ, Laudenslager ML, Mowat G, Heard D, Belant JL. Sex, diet, and the social environment: factors influencing hair cortisol concentration in free-ranging black bears (Ursus americanus). PLoS One. 2015 Nov; 10(11): $\mathrm{e} 0141489$.

29 First M, Williams JBW, Karg R, Spitzer R. Structured clinical interview for DSM-5. Research Version; 2015.

30 Rodakowski J, Skidmore ER, Reynolds CF, Dew MA, Butters MA, Holm MB, et al. Can performance on daily activities discriminate between older adults with normal cognitive function and those with mild cognitive impairment? J Am Geriatr Soc. 2014 Jul;62(7):1347-52.

31 Rogers J, Holm M. Performance assessment of self-care Skills (3.1). Pittsburgh, PA: 1984.

32 Jette AM, Haley SM, Coster WJ, Kooyoomjian JT, Levenson S, Heeren T, et al. Late life function and disability instrument: I. Development and evaluation of the disability component. J Gerontol A Biol Sci Med Sci. 2002 Apr;57(4):M209-16.

33 Karp JF, Skidmore E, Lotz M, Lenze E, Dew MA, Reynolds CF. Use of the late-life function and disability instrument to assess disability in major depression. J Am Geriatr Soc. 2009 Sep;57(9):1612-9.

34 Delis D, Kaplan E. Delis-Kaplan executive function system (DKEFS). San Antonio, TX: The Psychological Corporation; 2001.

35 Randolph C. Repeatable battery for the assessment of neuropsychological status. San
Antonio, TX: The Psychological Corporation; 1998.

36 Bora E, Harrison BJ, Yücel M, Pantelis C. Cognitive impairment in euthymic major depressive disorder: a meta-analysis. Psychol Med. 2013 Oct;43(10):2017-26.

37 Dybedal GS, Tanum L, Sundet K, Gaarden TL, Bjølseth TM. Neuropsychological functioning in late-life depression. Front Psychol. 2013 Jun; $4: 381$

38 Faust K, Nelson BD, Sarapas C, Pliskin NH. Depression and performance on the repeatable battery for the assessment of neuropsychological status. Appl Neuropsychol Adult. 2017 Aug;24(4):350-6.

39 Folstein MF, Folstein SE, McHugh PR. "Minimental state". A practical method for grading the cognitive state of patients for the clinician. J Psychiatr Res. 1975 Nov;12(3):189-98.

40 Miller MD, Paradis CF, Houck PR, Mazumdar S, Stack JA, Rifai AH, et al. Rating chronic medical illness burden in geropsychiatric practice and research: application of the $\mathrm{Cu}$ mulative Illness Rating Scale. Psychiatry Res. 1992 Mar;41(3):237-48.

41 Hulley S, Cummings S, Browner W, Grady D, Newman T. Designing clinical research: an epidemiological approach. 4th ed. Philadelphia, PA: Lippincott Williams \& Wilkins; 2013.

42 Dettenborn L, Muhtz C, Skoluda N, Stalder T, Steudte $\mathrm{S}$, Hinkelmann K, et al. Introducing a novel method to assess cumulative steroid concentrations: increased hair cortisol concentrations over 6 months in medicated patients with depression. Stress. 2012 May; 15(3):348-53.

43 Duncko R, Fischer S, Hatch SL, Frissa S, Goodwin L, Papadopoulos A, et al. Recurrence of depression in relation to history of childhood trauma and hair cortisol concentration in a community-based sample. Neuropsychobiology. 2019;78(1):48-57.

44 Gerritsen L, Staufenbiel SM, Penninx BWJH, van Hemert AM, Noppe G, de Rijke YB, et al. Long-term glucocorticoid levels measured in hair in patients with depressive and anxiety disorders. Psychoneuroendocrinology. 2019 Mar;101:246-52.

45 Hinkelmann K, Muhtz C, Dettenborn L, Agorastos A, Wingenfeld $\mathrm{K}$, Spitzer $\mathrm{C}$, et al. Association between childhood trauma and low hair cortisol in depressed patients and healthy control subjects. Biol Psychiatry. 2013 Nov; 74(9):e15-7.

46 Wei J, Sun G, Zhao L, Yang X, Liu X, Lin D, et al. Analysis of hair cortisol level in first-episodic and recurrent female patients with depression compared to healthy controls. J Affect Disord. 2015 Apr;175:299-302.

47 Pochigaeva K, Druzhkova T, Yakovlev A, Onufriev M, Grishkina M, Chepelev A, et al. Hair cortisol as a marker of hypothalamic-pituitary-adrenal axis activity in female patients with major depressive disorder. Metab Brain Dis. 2017;32(2):577-83. 